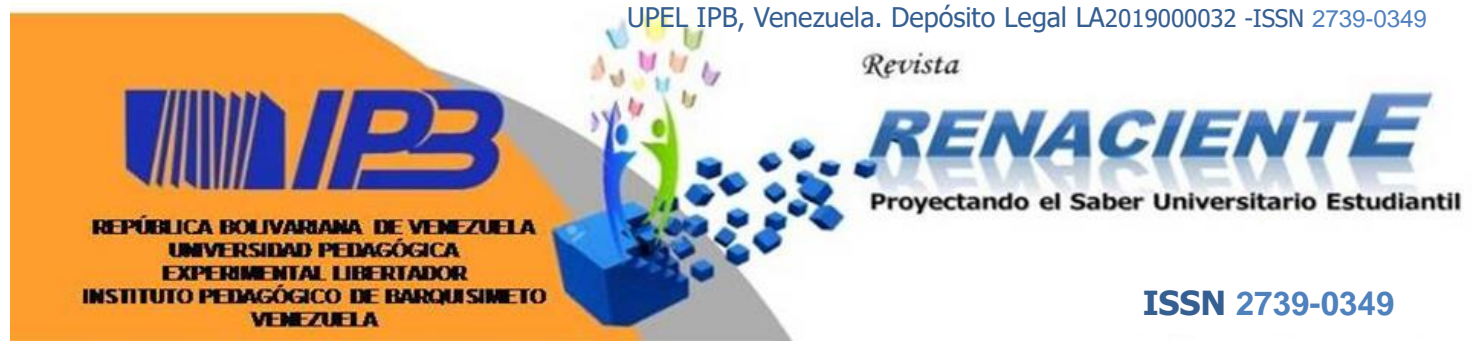

* Auqui Sislema Catty Maritza catty.auqui@unach.edu.ec https://orcid.org/0000-0001-6841-8399

* Chafla Tixi María Belén maria.chafla@unach.edu.ec https://orcid.org/0000-0001-8065$\underline{5223}$

* Chisaguano Conterón Mayita Pakaric pakaric.chisaguano@unach.edu.ec https://orcid.org/0000-0002-4697-1895

* Haro Mariño José Luis jose.haro@unach.edu.ec https://orcid.org/0000-0002-5536$\underline{2549}$

* Montoya LLuco Jimena Alexandra jimena.montoya@unach.edu.ec https://orcid.org/0000-0003-1003$\underline{7029}$

* Estudiantes de la Carrera de Pedagogía de las Ciencias Experimentales: Matemáticas y la Física de la Universidad Nacional de Chimborazo, perteneciente al Semillero de Trasposición Investigativa de la UNACH-Ecuador, Coordinado por la Dra. Carmen Varguillas.

** Docente Investigadora de la Universidad Nacional de Chimborazo y Coordinadora del Semillero de Trasposición Investigativa de la UNACH-Ecuador.

Recibido: 09-08-2021

Revisado: 15-09-2021

Aceptado: 30-09-2021

\section{EL APRENDIZAJE DE LAS MATEMÁTICAS A BASE DE LOS ERRORES ALGEBRAICOS}

\section{Resumen}

La presente investigación tiene el propósito de analizar el aprendizaje de la matemática en función de los errores algebraicos en la U.E "Velasco Ibarra" perteneciente al cantón Guamote, provincia de Chimborazo, del nivel de educación general básica. Se parte del planteamiento que el aprendizaje no es una facultad específica de los humanos, se dice que aprenden debido a la práctica o a la experiencia, para ello se define aprendizaje como proceso de habilidades y destrezas, el aprendizaje de las matemáticas es complejo, se dice tanto que los estudiantes como los docentes influyen en una decisión en el éxito del proceso de aprendizaje y enseñanza de las matemáticas, el teorema va más allá de un enunciado el significado de entender un teorema algebraico deberá darse en términos, los errores en las matemáticas no son cometidos solamente por los estudiantes si no por los docentes o matemáticos profesionales, los errores algebraicos son fuente de conocimiento que podemos explotar para profundizar en el pensamiento matemático.

Palabras clave: Aprendizaje, matemáticas, errores algebraicos

\section{THE LEARNING OF MATHEMATICS BASED ON ALGEBRAIC ERRORS}

Abstract

This is in order to indicate that learning is not a specific faculty of humans, it is said that they learn due to practice or experience, for this, learning is defined as a process of skills and abilities, learning mathematics is complex, it is said both that students and teachers influence a decision in the success of the process of learning and teaching mathematics, the theorem goes beyond a statement the meaning of understanding an algebraic theorem must be given in terms, the errors In mathematics they are not committed only by students but by teachers or professional mathematicians, algebraic errors are a source of knowledge that we can exploit to deepen mathematical thinking.

Keywords: learning, mathematics, algebraic errors 
Introducción

Los errores se encuentran en todos los niveles educativos, como en el desarrollo de la construcción del conocimiento matemático, se presentan de manera sistemática, en su desarrollo se debe considerar criterios diagnósticos, corrección y de tal manera el poder superar estos. Desde luego estos errores inciden en el aprendizaje de los contenidos, es muy indispensable que los estudiantes sepan y acojan la necesidad de superarlos para que de esta forma obtengan los logros dentro de su aprendizaje.

Numerosas investigaciones (Bednarz N, 1996)han puesto de manifiesto las dificultades que presenta el aprendizaje del álgebra en niveles preuniversitarios. Estas investigaciones, desde una perspectiva cognitivista, se centran en el análisis de los errores que tienen los alumnos cuando resuelven tareas algebraicas y en la asociación de estos errores a diferentes tipos de dificultades.

Con críticas constantes sobre la existencia de un error, podemos poner a prueba nuestro conocimiento diagnosticando, detectando, corrigiendo y corrigiendo el error. Los errores son datos objetivos que se encuentran a menudo en el proceso de enseñanza y aprendizaje de las matemáticas, por lo que este artículo fue creado para mostrar los errores algebraicos más comunes en los estudiantes de Séptimo semestre de la Carrera de las Ciencias Experimentales Matemáticas y Física de la Universidad Nacional de Chimborazo.

En la educación superior se dispone de un sistema educativo que busca alcanzar niveles de autonomía para los estudiantes durante el proceso de enseñanza aprendizaje. Desde tal enfoque, la universidad cuenta con la responsabilidad de definir los resultados académicos por cada nivel educativo por la que atraviesa el aprendiz. Esta autonomía refiere a la propia capacidad para obrar con libertar sin el necesario tutelaje de otra persona, por lo que implica además la obligación de hacerse responsable por los efectos que ocasiones sus decisiones (López, 2019) Pakaric, Haro Mariño José Luis, Montoya LLuco Jimena Alexandra : El aprendizaje de las matemáticas a base de los errores algebraicos 
El aprendizaje ha cobrado singular importancia en el espacio universitario, presentándose como requisito obligatorio para quienes estudian en la actualidad frente al reto creciente que la sociedad establece con un manejo de la información en flujo y cambio constante, lo que exige competencias más autónomas en el ejercicio del aprendizaje. Es por tal razón que autores como Cervera ( (Enríquez, 2014, pág. 4) afirman que las personas hoy en día no necesitan "información sino herramientas para poder ordenarla, sistematizarla y comprenderla, [pues la institución educativa] se ha de plantear su función no como facilitadora de procesos de aprendizaje sino como preparadora de procesos de autoaprendizaje. Por ello, la universidad realiza en su actividad educativa la facilitación del aprendizaje que promueva la autonomía y la toma de decisiones por el estudiante a partir de sus labores en el aula, en un proceso en conjunto en el que se aprende, se evalúan los logros y se comparten (Alfonzo, 2015)

\section{Argumentación Teórica}

\section{Aprendizaje}

El aprendizaje no es una facultad específica de los humanos, los animales se dice que aprenden debido a la práctica o a la experiencia, para ello se define aprendizaje como proceso o conjunto de procesos a través del cual o de los cuales, se adquieren o se modifican ideas, habilidades, destrezas, conductas o valores, como resultado o con el concurso del estudio, la experiencia, la instrucción, el razonamiento o la observación. (Zapata, s.f)

A su vez el aprendizaje es como un cambio relativamente permanente en la conducta o en su potencialidad que se produce a partir de la experiencia y que no puede ser atribuido a un estado temporal somático inducido por la enfermedad, la fatiga o las drogas. Esta definición contempla la experiencia como la condición esencial para el aprendizaje e incluye los cambios en la conducta. Desde el punto de vista del desarrollo del alumno, éste irá integrando sus conocimientos y destrezas a lo largo de la vida, en un proceso en el que 
intervienen las capacidades naturales, el nivel de madurez y el nivel de interacción con el medio. (Moralez, 2009)

\section{Aprendizaje de las matemáticas}

Se dice tanto que los estudiantes como los docentes influyen en una decisión en el éxito del proceso de aprendizaje y enseñanza de las matemáticas. Ambos son responsables por el desarrollo y los resultados de la práctica didáctica. Ambos tienen que aceptar sus ventajas y debilidades; ambos tienen que respetarse en sus formas de trabajar, aprender y enseñar. La responsabilidad por su propio aprendizaje y la enseñanza libre no significa la presencia y aceptación del desorden didáctico; por el contrario, requiere mayor atención por parte de alumnos y docentes.

También se dice que la didáctica crítica y progresista exige mayor acción en el proceso y mejor significado en el contenido, muy especialmente en el contenido matemático. Las dificultades con el aprendizaje de la matemática están ampliamente relacionadas con la poca acción que tienen los alumnos durante la realización de las actividades matemáticas. Estamos en presencia, entonces, de un problema didáctico, el cual puede ser resuelto mediante una concepción progresista de la pedagogía, tal como lo señaló claramente Paulo Freire.

Nos encontramos con un problema, en sentido estricto, si ante la presencia de una tarea o actividad desconocida requerimos de algunas reflexiones y consideraciones para poder suministrar coherentemente una solución satisfactoria. La enseñanza de las matemáticas, particularmente, está llena de situaciones inesperadas, lo cual podríamos señalar como un mundo desconocido transitado por interrogantes más que por soluciones o respuestas. No ocurre con frecuencia que los estudiantes suministren fácilmente soluciones directas a la variedad de problemáticas presentadas continuamente en las clases prácticamente en todas las asignaturas. Si esto ocurre, es porque los estudiantes están 
entrenados en la resolución de problemas o porque ellos reciben de parte de los docentes o del material de trabajo algunas sugerencias o indicaciones que les permiten encontrar una estrategia para la solución definitiva del respectivo problema. (Mora, 2003)

\section{Teoreoma}

Teorema es el sistema ternario conformado por un enunciado, la demostración de la relación de dependencia formulada en el enunciado, y el sistema teórico que la soporta. Esta definición de teorema va más allá de un enunciado y tiene en cuenta otros dos elementos. El significado de entender un teorema deberá darse en términos de los tres elementos. Así pues, se nombra a un teorema particular, el sistema teórico no solo sustenta la respectiva demostración, sino también cómo le da sentido matemático al enunciado, específicamente en lo que respecta al antecedente de la proposición condicional a través de la cual se expresa la relación de dependencia que se demuestra. (Molina, Samper, Perry, 2015)

\section{Pasos para entender un teorema}

La expresión a entender un teorema son los siguientes:

1. Identificar la estructura del enunciado, estructura desde el punto de vista de la lógica matemática.

2. Determinar el contenido geométrico del enunciado: identificar objetos y relaciones involucrados y atribuirles significado. Este aspecto permite establecer los contextos en los cuales es pertinente usar el teorema.

3. Realizar y entender la demostración de la relación de dependencia expresada en el enunciado.

a) Método de la demostración: determinar si es directa, indirecta haciendo uso del principio de reducción.

b) Estructura de la demostración: determinar los pilares de la demostración. Estos son: 
i) definiciones, postulados y teoremas.

ii) el propósito de usarlos en su desarrollo.

iii) la manera en que están involucrados en la demostración.

c) Prospectiva de la demostración: hacer un análisis con el fin de explorar o de predecir el comportamiento de un paso hipotético en la demostración.

4. Comparar con otros teoremas: la comparación se hace entre enunciados y entre demostraciones. La primera, consiste en determinar si los enunciados se refieren a asuntos semejantes. La segunda, que puede darse entre demostraciones de una misma relación de dependencia o de dos distintas, consiste en determinar si las demostraciones tienen una misma estructura o no. Específicamente, se trata de determinar si las demostraciones emplean elementos homólogos y, en caso de que así sea, si el propósito de hacerlo es análogo.

5. Usar de manera experta el teorema en diversos contextos: el propósito es determinar el contenido geométrico del enunciado de un teorema en donde se pueden utilizar. Reconocemos dos contextos en los que se puede usar un teorema:

i) En la justificación teórica de un procedimiento de construcción de un objeto geométrico con alguna propiedad especial, cuando se emplea como garantía de un paso en tal procedimiento.

ii) En la demostración de otro teorema, cuando se usa como garantía de una afirmación en un paso de la demostración. (Samper, 2015)

\section{Teoremas algebraicos}

Un teorema es un enunciado que puede ser demostrado como verdadero mediante operaciones matemáticas y argumentos lógicos. En matemáticas, un teorema es una proposición teórica, enunciado o formula que incorpora una verdad, axioma o postulado que es comprobada por otros conjuntos de teorías o formulas. Así mismo se lo cono como una regla o ley que se expresa en forma de ecuaciones o fórmulas matemáticas. (Ortega , 2016) 
En matemáticas el teorema fundamental del algebra superior, análisis matemático, geométrico y funciones de variable compleja, es un teorema que plantea que todo polinomio no constante de una variable y tiene al menos una raíz. Y se dice que se deriva que todo polinomio $\mathrm{p}(\mathrm{x})$ de una variable no constante tiene la misma cantidad de raíces reales o complejas que su grado $n$, resultado teórico que es vital para el cálculo numérico. (Salazar Cruz, 2009).

\section{Errores en Matemáticas}

Los errores en las matemáticas son cometidos solamente por los estudiantes y no por los docentes o matemáticos profesionales. Esta equivocada percepción en cuanto a quién comete errores o no durante el quehacer matemático ha contribuido con la mistificación del aprendizaje matemático. Saber matemáticas, se dice con frecuencia, es resolver los problemas o ejercicios matemáticos de manera independiente sin compartir con otros y cometer errores. Esta posición extrema asumida cotidianamente por muchos matemáticos y educadores matemáticos limita considerablemente el aprendizaje y provoca en los estudiantes un amplio rechazo hacia esta disciplina, aparecen en el trabajo de los alumnos, sobre todo cuando se enfrentan a conocimientos novedosos que los obligan a hacer una revisión o reestructura de lo que ya saben, los errores son intentos razonables pero no exitosos de adaptar a un conocimiento adquirido a una nueva situación entonces entendemos que el error va a tener distintas procedencias, pero siempre, se considera como un esquema cognitivo inadecuado y no solo como consecuencia de la falta de conocimiento o de un despiste. (Mora, 2003)

Los errores son defectos o averías que se producen durante el proceso de construcción del conocimiento o del desarrollo de las estructuras cognitivas, y son elementos usuales en el camino hacia el verdadero conocimiento, ya que al tratar de superarlos se pone en juego el ejercicio de la autocrítica, el sometimiento a prueba del conocimiento adquirido en diversos 
contextos y las diversas aproximaciones a la realidad construidas por el individuo. (Popper, 1979)

\section{Errores algebraicos}

Los errores que tienen su origen en una ausencia de sentido se originan en los distintos estadios de desarrollo (semiótico, estructural y autónomo) que se dan en los sistemas de representación, por lo que podemos diferenciar en tres etapas distintas. (Ruano, Socas, \& Palarea, 2001)

1) Errores de algebra que tiene su origen en el Aritmética, para entender la generalización de las relaciones y procesos se requiere que estos antes hayan sido asimilados en el contexto aritmético.

2) Errores de procedimiento de que los alumnos usan inapropiadamente formulas o reglas de procedimiento.

3) Errores de algebra debido a las características propias del lenguaje algebraico.

El problema de la enseñanza y el aprendizaje del álgebra es un tema de interés general y que no ha sido resuelto en su totalidad hasta la actualidad, más específicamente, los errores que exhiben los estudiantes al resolver distintas tareas de carácter algebraico son una clara evidencia de las dificultades que manifiestan al enfrentarse a este tipo de actividades.

Se dice que los errores tienen su origen en actitudes afectivas y emocionales tiene distinta naturaleza: falta de concentración, bloqueos, olvidos, etc.

El origen de los errores fue Socrates, a traves de su doctrina de la factibilidad en la que proponia que el hombre que puede errar individual y colectivamente debe aspirar a la verdad objetiva examinando sus errores mediante la autocritica y la critica racional este doctrina no debe ser considerada como una idea de epistemologia pesimista ya que como se explico, implica que el hombre puede buscar la verdad, la verdad objetiva, aunque en muchos casos se puede equivocar por amplio margen; esta idea socratica agrega que si se 
respeta la verdad, se debe aspirar a ella examinando persistemente los errores cometidos, siendo criticos y autocriticos. (Garcia, 2016).

Una reflexión general acerca del origen de las dificultades que presentan los estudiantes en el aprendizaje de las matemáticas y las clasifican en cinco categorías. Las dos primeras tienen relación con la disciplina: la complejidad de los objetos matemáticos por un lado y los procesos de pensamiento matemático por otro. La tercera categoría se centra en el proceso de enseñanza desarrollado para el aprendizaje de las matemáticas, y tiene relación con los materiales curriculares, los recursos y los estilos de enseñanza. La cuarta categoría se asocia al proceso del desarrollo cognitivo del estudiante, en el cual se toman en consideración varios enfoques de aprendizaje. Finalmente, existe un grupo de dificultades asociadas a actitudes afectivas y emocionales hacia la matemática, las cuales se vinculan con la ansiedad y el miedo hacia la asignatura, en muchas ocasiones el estudiante desarrolla cierta resistencia al dejar de cometer el error: es decir, la corrección de los errores implica, en ocasiones, corregir un conocimiento aprendido erróneamente. Además, agrega que los errores pueden generarse por una comprensión equivocada, que el estudiante considera correcta o errores al azar, que reflejan falta de cuidado. Es decir, cuando un contenido matemático no es significativo para el estudiante y carece de sentido para él, en ocasiones, se puede evidenciar en los errores que presenta el estudiante al resolver ejercicios matemáticos. (Socas, 2016).

\section{Metodología.}

El proceso de investigación se abordó desde un enfoque cuantitativo. Es de tipo descriptiva porque permitió determinar la característica fundamental de la investigación y los involucrados en el proceso al igual es de tipo documental porque la investigación se realizará mediante la consulta de documentos (libros, revistas, periódicos, memorias, anuarios, registros, códices, constituciones, etc.). 


\section{Nivel de investigación}

Descriptivo: Porque se describirá los datos y características de la población o fenómeno en estudio.

Según (Sabino, 2012), la investigación de tipo descriptiva trabaja sobre realidades de hechos, y su característica fundamental es la de presentar una interpretación correcta. Para la investigación descriptiva, su preocupación primordial radica en descubrir algunas características fundamentales de conjuntos homogéneos de fenómenos

El test fue aplicado en la U.E "Velasco Ibarra" perteneciente al cantón Guamote, provincia de Chimborazo, en la cual su población está constituida por los estudiantes de 10 año "A" de educación general básica, constituido por 32 estudiantes, los cuales son 15 hombres y 17 mujeres, teniendo la mayoría de estudiantes 12 años de edad y siendo una gran parte de etnia indígena

\section{Técnicas e instrumentos de Recolección de la información}

Técnica: se utilizó la encuesta como técnica para la recolección de la información, el cual será de ayuda para elaborar datos de modo rápido y eficaz

Instrumento: Se utilizó el Test Psicopedagógico con preguntas cerradas, ya que las posibles respuestas son mutuamente excluyentes, es decir fácil de tabular y codificar, al igual que fáciles de aplicar

Técnicas de análisis : Para el procesamiento de análisis e interpretación de datos se usará en el paquete informático de Microsoft Office Excel, mediante técnicas de estadística descriptiva.

\section{Análisis e interpretación de resultados}

A continuación, se presentan los resultados obtenidos del instrumento de recolección de información, en función de los ítem que lo conformaban. 


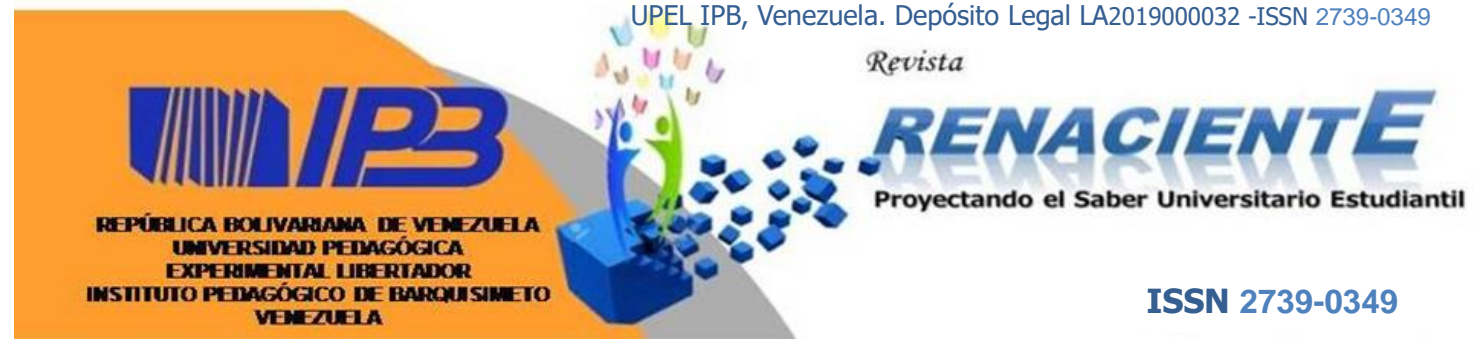

Item 1.-Me siento preparado para las clases de matemáticas

$\begin{array}{lll} & \text { N. Personas } & \text { \% } \\ \text { Siempre } & 19 & 59.4 \% \\ \text { Casi siempre } & 7 & 21.9 \% \\ \text { A veces } & 6 & 18.8 \% \\ \text { Nunca } & 0 & 0 \% \\ \text { Total } & \mathbf{3 2} & \mathbf{1 0 0 \%}\end{array}$

Análisis: El 59.40\% de encuestados mencionan que se encuentran preparados para las clases de matemáticas mientras que el $21.90 \%$ y el $18.80 \%$ manifiestan que casi siempre y a veces respectivamente.

Interpretación: Se puede evidenciar que la mayoría de los encuestados se encuentran siempre preparados para las clases de matemáticas.

Item 2.-Tengo confianza en lograr buenas notas en matemáticas.

$\begin{array}{lll} & \text { N. Personas } & \text { \% } \\ \text { Siempre } & 16 & 50 \% \\ \text { Casi siempre } & 10 & 31.3 \% \\ \text { A veces } & 6 & 18.8 \% \\ \text { Nunca } & 0 & 0 \% \\ \text { Total } & \mathbf{3 2} & \mathbf{1 0 0 \%}\end{array}$

Análisis: El 50\% de encuestados mencionan que siempre tienen confianza de lograr buenas notas en matemáticas mientras que el $31.10 \%$ y el $18.80 \%$ de encuestados mencionan que casi siempre y a veces tienen confianza en lograr buenas notas en matemáticas.

Interpretación: Se puede observar que la mayor parte de encuestados manifiestan que siempre tienen confianza de lograr buenas notas en matemáticas. 


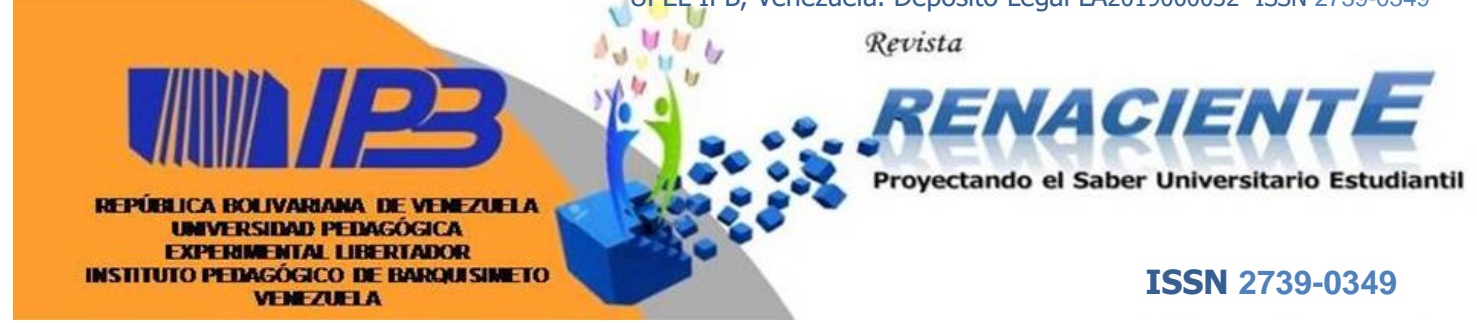

Item 3.-Siento que mis padres confían en que me va a ir bien en matemáticas.

$\begin{array}{lll} & \text { N. Personas } & \text { \% } \\ \text { Siempre } & 21 & 65.6 \% \\ \text { Casi siempre } & 4 & 12.5 \% \\ \text { A veces } & 7 & 21.9 \% \\ \text { Nunca } & 0 & 0 \% \\ \text { Total } & \mathbf{3 2} & \mathbf{1 0 0 \%}\end{array}$

Análisis: El 65.60\% de encuestados mencionan que sienten que sus padres confían en que les va ir bien en matemáticas, mientras que el $21.90 \%$ y el $12.50 \%$ que a veces y casi siempre sienten que sus padres confían en que les va ir bien en matemáticas.

Interpretación: Se puede evidenciar que la mayor parte de encuestados mencionan que siempre sienten que sus padres confían en que les va a ir bien en matemáticas.

\section{Item 4.-Me gustan las clases de matemáticas}

$\begin{array}{lll} & \text { N. Personas } & \% \\ \text { Siempre } & 21 & 65.6 \% \\ \text { Casi siempre } & 8 & 25 \% \\ \text { A veces } & 3 & 9.4 \% \\ \text { Nunca } & 0 & 0 \% \\ \text { Total } & \mathbf{3 2} & \mathbf{1 0 0 \%}\end{array}$

Análisis: El 65.60\% de encuestados menciona que siempre les gusta las clases de matemáticas mientras que el $25 \%$ y $9.40 \%$ menciona que casi siempre y a veces les gustan las clases de matemáticas.

Interpretación: Se puede observar que la mayor parte de encuestados manifiestan que siempre les gustan las clases de matemáticas. 


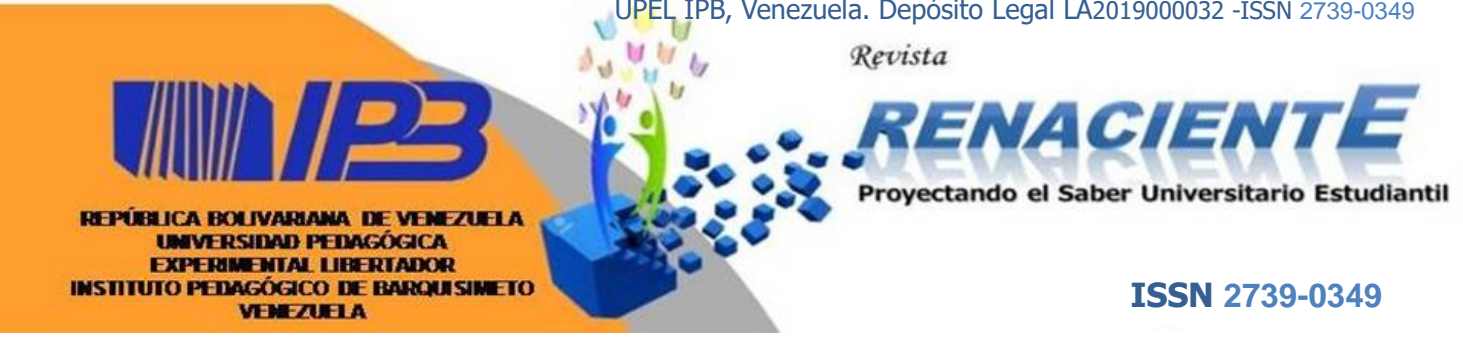

Item 5.-Intentó corregir mis errores en matemáticas

\section{N. Personas \%}

$\begin{array}{lll}\text { Siempre } & 25 & 78.1 \% \\ \text { Casi siempre } & 6 & 18.8 \% \\ \text { A veces } & 1 & 3.1 \% \\ \text { Nunca } & 0 & 0 \% \\ \text { Total } & \mathbf{3 2} & \mathbf{1 0 0 \%}\end{array}$

Análisis: El 78.10\% de los encuestados muestran que siempre intentan corregir sus errores en matemáticas mientras que el $18.80 \%$ y $3.10 \%$ mencionan que casi siempre y a veces intentan corregir sus errores en matemáticas.

Interpretación: Se observa que la mayor parte de encuestados siempre intentan corregir sus errores en matemáticas.

\section{Discusión}

Luego de obtener los resultados de la investigación se pudo lograr identificar y analizar información muy importante en cuanto al nivel de afectividad hacia la matemática, en donde se pudo constatar que los estudiantes manejan un nivel afectivo positivo ya que se muestra que se esfuerzan por mejorar su aprendizaje y esto va relacionado con la confianza que les muestran sus docentes y compañeros de clases en el desarrollo de su aprendizaje matemático. Ello permite determinar que muestran para estar preparados ante cualquier situación lo que menciona (Mora,2003) tanto los estudiantes como los docentes influyen determinantemente en el éxito del proceso de aprendizaje y enseñanza de las matemáticas. Ambos son responsables por el desarrollo y los resultados de la práctica didáctica. Ambos tienen que aceptar sus ventajas y debilidades; ambos tienen que respetarse en sus formas de trabajar, aprender y enseñar. 
También tomando en cuenta lo que menciona McLeod que se inclina por incluir las creencias, las emociones y las actitudes como factores básicos para dominio de la afectividad, que se relaciona con lo que se presentó como interrogantes en el test aplicado donde se pudo constatar que los estudiantes prestan atención a las clases de matemáticas y no se sienten presionados al momento de realizar evaluaciones o pruebas ya que manejan una actitud positiva, están preparados y seguros en obtener buenos resultados lo que es promovido por sus docentes, padres de familia y compañeros lo que garantiza su éxito en el aprendizaje matemático.

\section{Conclusión}

Con el análisis realizado en la investigación sobre la afectividad hacia las matemáticas de los estudiantes de octavo año de educación básica de la Unidad Educativa Velasco Ibarra, se evidencia que se encuentra en un nivel medio, tal como refleja en los resultados de la encuesta realizada, debido a que, denotan no ser siempre las situaciones en las que se sienten bien frente a las matemáticas.

\section{Referencias}

CC.OO, F. d. (2009). Temas para la educación.

(6). https://www.feandalucia.ccoo.es/plantillai.aspx?p=5\&d=6752

Cosacov. (2010). La afectividad. s.m.r. https://portalacademico.cch.unam.mx/repositorio-desitios/experimentales/psicologia1/Ps1/MD3/MD3-

\section{L/LECTURA_AFECTIVIDAD.pdf}

Erazo, J. y Aldana, E. (2015) Sistema de creencias sobre las matemáticas en los estudiantes de educación básica. Revista Praxis https://documat.unirioja.es/descarga/articulo/5907253.pdf

Garcia, F., Fonseca, G., \& Concha, L. (2015). Aprendizaje y rendimiento académico en educación superior: un estudio comparado Revista Electrónica "Actualidades 


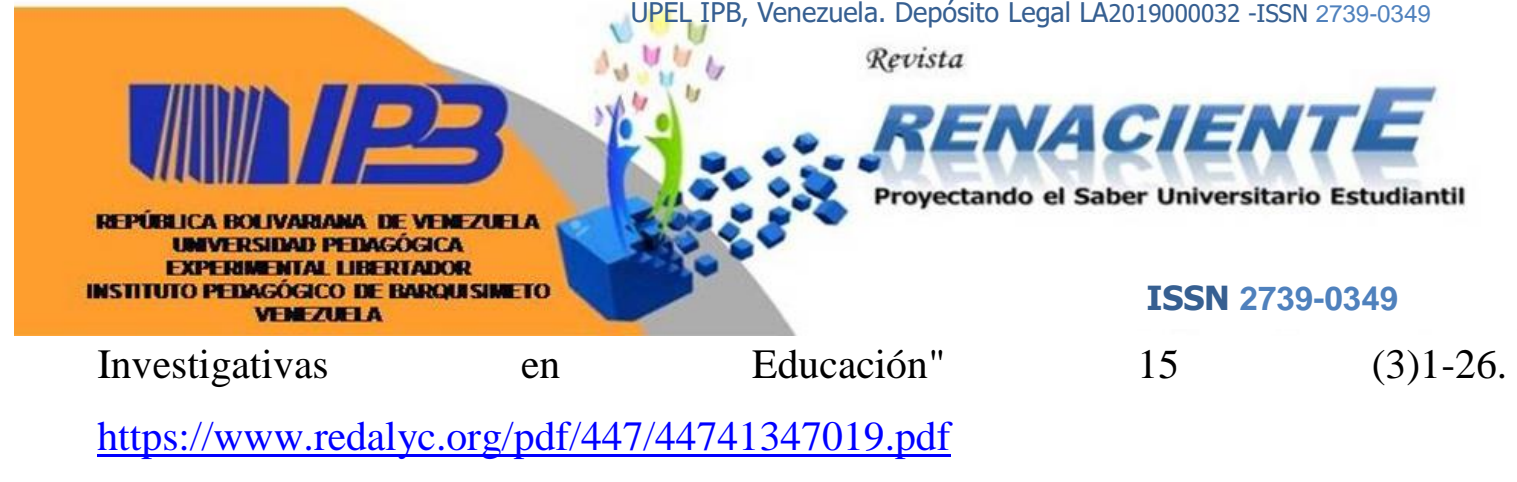

Gil, N., Guerrero, E., \& Blanco, L. (2006). El dominio afectivo en el aprendizaje de las matemáticas. Electronic Journal of Research in Educational Psychology, 4 (1) 47-72 https://www.redalyc.org/pdf/2931/293123488003.pdf

Martínez, O. (2015) Dominio afectivo en educación matemática. Paradígma, 26(2), 7-34. http://ve.scielo.org/scielo.php?script=sci_arttext\&pid=S1011-22512005000200002

Mora, C. (2003). Estrategias para el aprendizaje y la enseñanza de las matemáticas. Estrategias para el aprendizaje y la enseñanza de las matemáticas. Revista de Pedagogía, 24(70), 181-272. http://ve.scielo.org/scielo.php?script=sci_arttext\&pid=S0798-97922003000200002

Morales, A. (2019) Toda Materia. Blog. Recuperado el 22 de Marzo de 2021, de https://www.todamateria.com/aprendizaje/

Sabino, C. (2012). Investigación descriptiva. Tesisplus. Obtenido de https://tesisplus.com/investigacion-descriptiva/investigacion-descriptiva-segunautores/ Pakaric, Haro Mariño José Luis, Montoya LLuco Jimena Alexandra : El aprendizaje de las matemáticas a base de los errores algebraicos 